\title{
Estimating Topographic Blocking Using a Froude Number When the Static Stability Is Nonuniform
}

\author{
Patrick A. Reinecke And Dale R. Durran \\ Department of Atmospheric Sciences, University of Washington, Seattle, Washington
}

(Manuscript received 13 March 2007, in final form 19 June 2007)

\begin{abstract}
A parameter widely used to predict topographic flow blocking is the nondimensional mountain height or, synonymously, the inverse Froude number. Predictions using this parameter are based on the morphology of flows with uniform upstream static stability and wind speed, which rarely occur in the real world. The appropriateness of applying this theory in the presence of nontrivial background stability is therefore investigated using a numerical model. Two methods were considered to estimate the low-level stability, averaging the Brunt-Väisälä frequency below the crest and using the bulk change in $\theta$ between the ground and crest level.

No single best method emerged for estimating the upstream static stability and thereby mapping the simulations with inversions onto the set of solutions with constant stratification. Instead, the best method depended on the application at hand. To predict the onset of flow stagnation, averaging the low-level stability worked best, while to predict low-level flow diversion the bulk estimate of low-level stability was most appropriate. These results are consistent across a range of inversion thicknesses and strengths. In addition, it is shown that variations in static stability above the mountain crest have little impact on flow blocking.
\end{abstract}

\section{Introduction}

The ability to determine if flow impinging a mountain freely rises over the mountain or is blocked and deviates around the mountain is of great practical importance in regions of complex terrain. In addition to the impact on pollution dispersion, barrier and flank jets in the vicinity of the massif are also associated with blocked flow (e.g., Marwitz 1983; Georgelin and Richard 1996). The transition from flow over the crest to blocked flow may also reduce the amplitude of lee waves generated by the massif, which in turn may reduce the wave drag by reducing the height of the topography that the unblocked portion of the flow encounters. Stagnant air generated by blocked flow may also absorb downward-propagating trapped lee waves, which leads to lee-wave decay (Smith et al. 2002). In addition, ridge-parallel variations in the blocked flow can lead to increased precipitation on the windward

Corresponding author address: Patrick A. Reinecke, Department of Atmospheric Sciences, University of Washington, Box 351640, Seattle, WA 98105-1640.

E-mail: reinecke@atmos.washington.edu side by enhancing low-level convergence (Rotunno and Ferretti 2001).

A relatively simple theory introduced by Smith (1988) for circular mountains and extended by Smith (1989) to include elliptical mountains with varying horizontal aspect ratio, $\beta=b / a$, estimates the conditions under which blocking occurs using linear theory for airflow over an isolated mountain in which both the crossmountain wind speed $U$ and Brunt-Väisälä frequency $N$ are constant upstream. We refer to this as the constant $U$ and stratification (CUS) theory. Neglecting Coriolis forces and assuming the motions are incompressible, hydrostatic, and steady, one may show that the flow is completely determined by two parameters: $\beta$ and $\epsilon=N h_{0} / U$, the mountain height normalized by a scale for the vertical wavelength of a linear $2 \mathrm{D}$ hydrostatic mountain wave. The parameter $\epsilon$ has been referred to as the inverse Froude number since it is the ratio of a wave speed to the flow speed; however, more appropriately, it can be thought of as describing the nonlinearity of the flow since the perturbation $u$ in the linear limit is proportional to $N h_{0}$. Smith (1989) found that, as $\epsilon$ increases, stagnation in the fluid first occurs on the windward slope for mountains elongated in the 
direction of the flow $(\beta<1)$ and occurs first above the lee slope in the form of wave breaking for mountains elongated in the cross-flow direction $(\beta>1)$. Numerical studies by Smolarkiewicz and Rotunno (1990), Ólafsson and Bougeault (1996), and Bauer et al. (2000) have confirmed the general shape of Smith's curve for stagnation.

In addition to providing insight into the dynamics of flow blocking, the simplicity of the CUS theory provides an attractive method to diagnose actual atmospheric states as either blocked or unblocked. The theory is, however, directly applicable only to very idealized atmospheric structures, which are often quite different from those observed in actual atmospheric events. To employ the CUS theory, the actual atmospheric structure must be simplified by estimating an effective constant cross-mountain wind speed $U$ and by approximating the actual stability profile as constant with height. Two methods of approximating the constant static stability have appeared frequently in the literature. In one method, the Brunt-Väisälä frequency is averaged between the ground and some height aloft to yield a constant $N$ (e.g., Mass and Ferber 1990; Schumacher et al. 1996; Bénech et al. 1998; Medina and Houze 2003; Jiang et al. 2005). We refer to this as the averaging method. In the other method the total change of the potential temperature between the ground and some level aloft is used to define $N$ (e.g., Manins and Sawford 1982; Chen and Smith 1987; Spangler 1987; Overland and Bond 1995; Kalthoff et al. 2002). We refer to this as the bulk method. The goal of this paper is to evaluate how well the CUS theory predicts blocked flow when such estimates are used.

Ideally one would like a simple theory for upstream blocking that could be accurately applied to finiteamplitude situations with arbitrary vertical profiles of wind speed and stability, but no such theory currently exists. Therefore, we will test the CUS model on a more restricted class of problems in which the velocity of the undisturbed cross-mountain flow is uniform, but the static stability profile contains significant variations in the form of a near-mountaintop stable layer capping a weakly stratified layer near the ground. Typical deep tropospheric values for the static stability will be specified above the inversion. This type of static stability profile is chosen for two reasons. First, this structure represents a maximum realistic perturbation about the constant- $N$ profiles assumed in CUS theory; the performance of both the averaging and bulk methods improve as the variations in the low-level stability become more gradual, so cases involving elevated inversions provide the most discriminating test. Second, such inversions are present in many important atmospheric applica- tions; situations in which a weakly stratified layer capped by a near-mountaintop stable layer impinged on a mountain barrier have been observed in the Rocky Mountains (Brinkmann 1974), the Sierras (Marwitz 1983), and the Alps (Bousquet and Smull 2003). In addition, upstream blocking and lee vortex formation produced by mountainous islands often occurs when strong inversions capping a layer of weak static stability are present below mountaintop level (Schär and Smith 1993).

In section 2, we begin by describing the strategies for approximating the actual static stability profile to obtain the parameters required for application of the CUS theory. The numerical model is also described in section 2. Section 3 is devoted to examining the morphological differences between cases with and without inversions. In section 4 we consider two ways to characterize the degree of blocked flow: the minimum wind speed on the windward slope and the percent of mass initially upstream of the mountain that deviates laterally around the mountain. We also examine whether the bulk method or the averaging method is better suited to estimate the low-level stability. The sensitivity of our results to the stability above mountain top is investigated in section 5. Finally, in section 6 we present our conclusions.

\section{Experimental setup}

\section{a. Estimating soundings}

To apply the CUS theory to a sounding with nonuniform Brunt-Väisälä frequency, some method to estimate the static stability must be employed. We consider two such methods: an average stability estimate and a bulk stability estimate. In the averaging method the low-level stability is estimated as

$$
N_{A}=\frac{1}{h_{0}} \int_{0}^{h_{0}} N(z) d z
$$

In the bulk method the total change of $\theta$ between the ground and $h_{0}$ is used to characterize the stability,

$$
N_{B}=\sqrt{\frac{g}{\theta_{00}} \frac{\theta_{h_{0}}-\theta_{00}}{h_{0}}},
$$

where $\theta_{00}$ is the reference potential temperature at the ground and $\theta_{h_{0}}$ is the potential temperature at $h_{0}$. This is equivalent to averaging $N^{2}$ between 0 and $h_{0}$ and taking the square root.

\section{b. Upstream soundings}

All soundings contain an inversion at or below the level of the mountain crest. Figure 1 shows the $\theta$ profile 


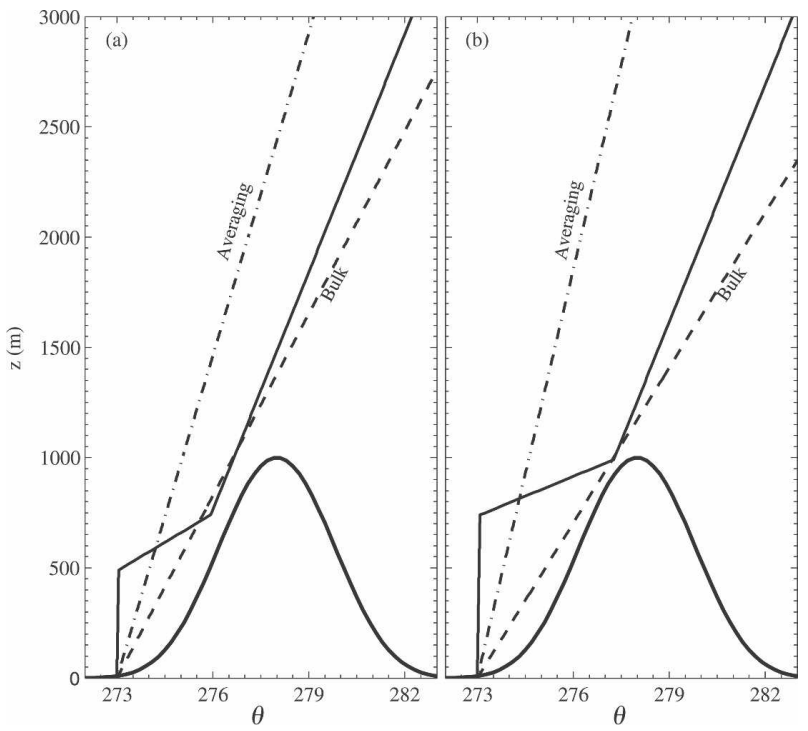

FIG. 1. Upstream soundings of $\theta$. Solid line indicates the actual sounding for the (a) below-mountaintop inversion and (b) mountain-top inversion for the case $h_{0}=1000 \mathrm{~m}$. The dashed and dashdot lines are the $\theta$ profiles obtained with the bulk and averaging methods of the stability below the level of the mountain top.

of two such soundings. In this first set of soundings, the inversions will be described by the position of their tops relative to the top of the mountain. The layer is either "below mountain top" (inversion centered at $5 / 8 h_{0}$, Fig. 1a) or "at the "mountain top" (inversion centered at $7 / 8$ $h_{0}$; Fig. 1b). The stability is constant in all three layers and independent of $h_{0}$. Like the inversion height, the inversion thickness in our first set of experiments also scales with $h_{0}$ and is $1 / 4 h_{0}$. This scaling minimizes the number of control parameters by ensuring that $N_{A}$ and $N_{B}$ are independent of $h_{0}$. The Brunt-Väisälä frequencies above and below the inversion are $N_{U}=0.010 \mathrm{~s}^{-1}$ and $N_{L}=0.002 \mathrm{~s}^{-1}$, respectively. Apparent in Fig. 1 is that vertical profiles with constant $N_{A}$ underestimate the Brunt-Väisälä frequency aloft, whereas those with constant $N_{B}$ overestimate it. To minimize the magnitude of the deviation of $N_{A}$ and $N_{B}$ from the reference profile the Brunt-Väisälä frequency within the inversion is set to $N_{I}=0.020 \mathrm{~s}^{-1}$ for the below-mountaintop inversion and $N_{I}=0.025 \mathrm{~s}^{-1}$ for the mountaintop inversion.

A second set of soundings are also considered in which the height of the center of the inversion is held fixed while its thickness and the mountain height are varied. The $\theta$ profiles, as well as the mountain heights for these simulations, are shown in Fig. 2. Three inversion thicknesses are considered: 125,250 , and $500 \mathrm{~m}$, with stabilities of $N_{I}=0.035 \mathrm{~s}^{-1}, N_{I}=0.025 \mathrm{~s}^{-1}$, and $N_{I}=0.017 \mathrm{~s}^{-1}$, respectively. These stabilities are cho-

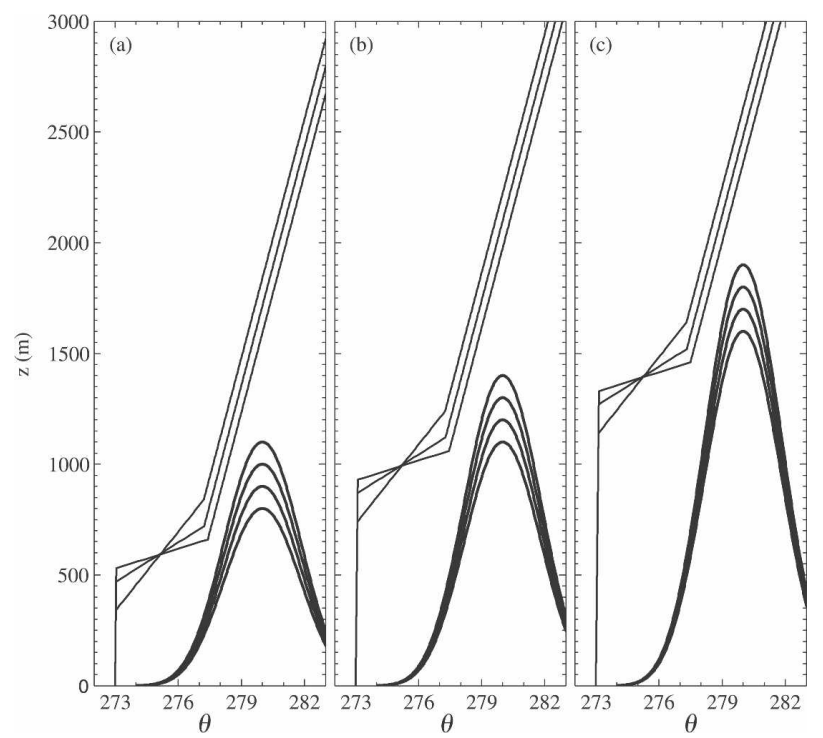

FIG. 2. Upstream soundings of $\theta$ for the second set of soundings considered. The middle of the inversion is fixed at (a) $600 \mathrm{~m}$, (b) $1000 \mathrm{~m}$, and (c) $1400 \mathrm{~m}$. Three inversion thickness are considered 125,250 , and $500 \mathrm{~m}$. The four mountain heights for each experiment are also shown.

sen so that the increase in the potential temperature across the inversion is identical in all cases. These additional soundings provide further test for both the averaging and bulk methods.

\section{c. Numerical model}

No analytic solution exists for the finite-amplitude flow of a constant $N$ and $U$ atmosphere around a 3D mountain; therefore, we must turn to a numerical model to evaluate the flow behavior. We also use the numerical model to simulate the cases with inversions in the upstream flow. The numerical model follows Durran and Klemp (1983) and Epifanio and Durran (2001). The model is based on the inviscid, threedimensional, nonrotating, compressible Boussinesq equations of motion. It is fully nonlinear, nonhydrostatic and employs a free-slip lower boundary. The firstorder closure scheme of Lilly (1962) is used to parameterize subgrid-scale turbulence. At the top boundary a linear radiation condition based on Klemp and Durran (1983) and Bougeault (1983) and modified by Durran (1999) for local evaluation is used. To prevent unphysical maxima and minima from developing in the $\theta$ field near the edges of the inversion a flux-limited advection scheme (LeVeque 1996) is used to advect $\theta$ in cases with inversions. A more economical centered fourthorder advection scheme is used in all of the cases with constant upstream stability.

The topography used in the model is an elongated 
TABLE 1. Values of $h_{0}$, inversion heights, and inversion thicknesses for each value of $\beta$ used in this study.

\begin{tabular}{lccc}
\hline \hline & $\begin{array}{c}\text { Inversion } \\
\text { thickness }(\mathrm{m})\end{array}$ & $\begin{array}{c}\text { Inversion } \\
\text { height }(\mathrm{km})\end{array}$ & $\mathrm{h}_{0}(\mathrm{~km})$ \\
\hline 1 & $1 / 4 h_{0}$ & $5 / 8 h_{0}$ & $1.4,1.6,1.8,2.0$ \\
1 & $1 / 4 h_{0}$ & $7 / 8 h_{0}$ & $1.8,2.0,2.2,2.4$ \\
2,4 & $1 / 4 h_{0}$ & $5 / 8 h_{0}$ & $0.8,1.0,1.2,1.4$ \\
2,4 & $1 / 4 h_{0}$ & $7 / 8 h_{0}$ & $1.0,1.2,1.4,1.6$ \\
2 & $125,250,500$ & 0.6 & $0.8,0.9,1.0,1.1$ \\
2 & $125,250,500$ & 1.0 & $1.1,1.2,1.3,1.4$ \\
2 & $125,250,500$ & 1.4 & $1.6,1.7,1.8,1.9$ \\
\hline
\end{tabular}

ridge with a uniform-height center section, used by Epifanio and Durran (2001), defined as

$$
h(x, y)=\left\{\begin{array}{cl}
\frac{h_{0}}{16}[1+\cos (\pi r)]^{4}, & \text { if } \quad r \leq 1 \\
0, & \text { otherwise }
\end{array}\right.
$$

where

$$
r^{2}=\left\{\begin{array}{cll}
\left(\frac{x}{4 a}\right)^{2}+\left[\frac{|y|-(\beta-1) a}{4 a}\right]^{2}, & \text { if } & |y|>(\beta-1) a \\
\left(\frac{x}{4 a}\right)^{2}, & \text { otherwise. }
\end{array}\right.
$$

The different mountain heights, inversion heights, and inversion thicknesses are summarized in Table 1. The undisturbed upstream wind speed is $U=10 \mathrm{~m} \mathrm{~s}^{-1}$. The flow is in the hydrostatic limit because $a$ is chosen such that $N_{u} a / U=10$ where $N_{U}$ is the stability aloft. To limit spurious gravity waves the model is initiated by gradually increasing $u$ from rest to $U$ over a time interval $-4 \leq U a / t \leq 0$. We run the model until $U t / a=25$, at which time the upstream flow features are nearly in steady state.

The three nested grids shown in Fig. 3 are used in all simulations to make the integration more computationally efficient. The location of the upper-right and lowerleft corners of each nest relative to the mountain center, located at the origin, are also shown. The largest nest extends $36 a$ in each direction from the mountain center. The second finest mesh extends $-12 a$ upstream and $18 a$ downstream of the mountain center. The lateral edges of the second finest mesh are $\pm 15 a$ from the mountain center. The finest mesh extends $-5 a$ upstream and $15 a$ downstream with the lateral edges lying $\pm 7 a$ from the mountain center. The horizontal grid spacing is $\Delta=$ $0.1 a$ on the finest mesh and increases by a factor of 3 for each larger mesh. The vertical grid spacing is $\Delta z=$ $h_{0} / 32$ for $z<3 / 2 h_{0}$ and then stretches to $\Delta z=U /\left(4 N_{U}\right)$. This ensures that the inversion will be well resolved with at least eight grid points while allowing the inte-

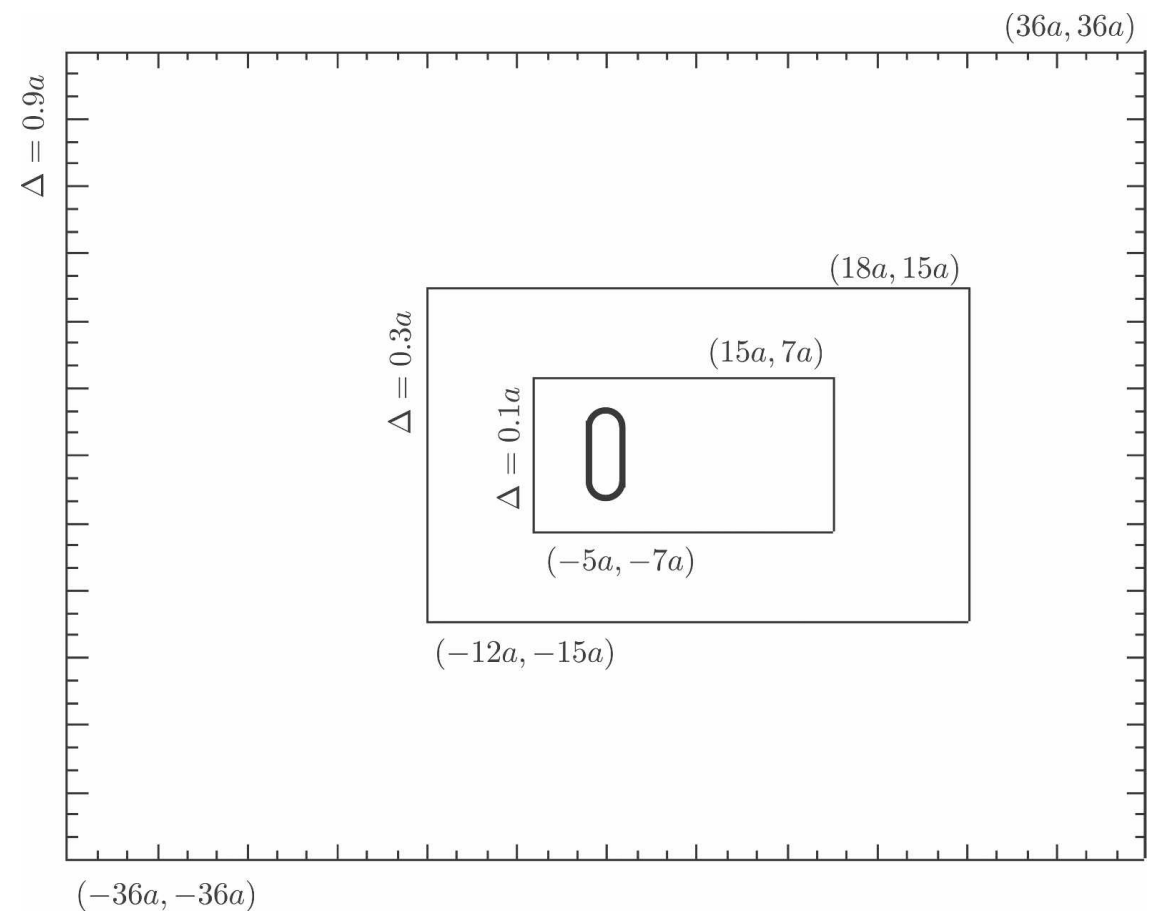

FIG. 3. Location and size of the three nested grids. The values at the upper-right and lower-left corners are distances from the corner to mountain center. 
gration to be computationally efficient. The top boundary is

$$
z_{t}=\frac{9}{2} \frac{U}{N_{U}} \pi
$$

To not violate the CFL condition the large time step on the finest mesh is $\Delta t=0.025 \mathrm{a} / \mathrm{U}$ and increases by a factor of 3 on each larger mesh.

\section{Flow morphology with varying upstream soundings}

In this section the qualitative behavior of the solution with an upstream inversion is compared to the corresponding solutions from CUS theory obtained using both averaging and bulk methods.

\section{a. Upstream structure}

Figure 4a shows the $u$ component of velocity and $\theta$ contours in an $x-z$ cross section along the centerline of the mountain at a nondimensional time of $U t / a=25$ for a simulation in which $\beta=4, h_{0}=1400 \mathrm{~m}$, and the upstream sounding is the below-mountaintop inversion profile shown in Fig. 1a. Note that, due to upstream influence, the inversion has been displaced about $300 \mathrm{~m}$ upward at the left edge of the figure. Figure 4a shows that upstream of $x=-1.9 a$ the flow field below the inversion is relatively independent of $z$. Downstream of $x=-1.9 a$ a region of reversed flow extends horizontally to $x=-0.9 a$ where it intersects the mountain. Figure 5a shows the $x-y$ distribution of the $u$ component of surface wind speed for the same belowmountaintop inversion case shown in Fig. 4a. The region of reversed flow is marked by the heavy white contour and runs laterally along the mountain slope to $y= \pm 3.2 a$ which is slightly beyond the uniform-height section of the ridge.

Figures $4 \mathrm{~b}$ and $5 \mathrm{~b}$ show numerical simulations for a case identical to that in Figs. 4a and 5a except that the upstream static stability profile is constant with the $\theta$ profile given by the averaging method shown (dashdotted line in Fig. 1a). In this case the nonlinearity parameter is

$$
\epsilon_{A}=\frac{N_{A} h_{0}}{U}=1.14
$$

Note that the flow reverses further upstream than in the below-mountaintop inversion case and there is more vertical wind shear below $z=1 \mathrm{~km}$.

A third simulation, identical to the first two except that the upstream thermodynamic profile is that given by the bulk method (dashed line in Fig. 1a), is shown in
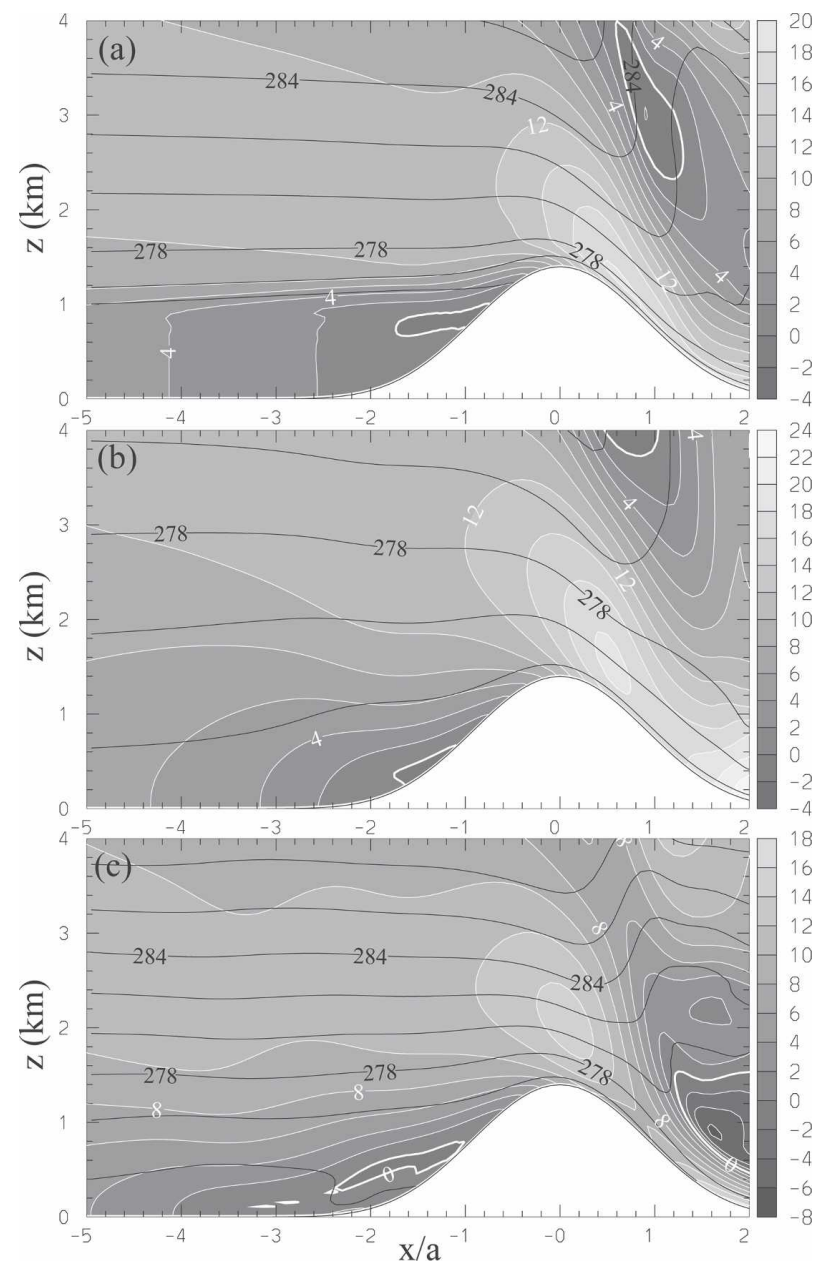

FIG. 4. The $u$ component of the wind along the centerline $y=$ 0 , shown in shading and white contours at $U t / a=25$ for a $h_{0}=$ $1400 \mathrm{~m}, \beta=4$ mountain and an upstream wind speed of $U=10$ $\mathrm{m} \mathrm{s}^{-1}$. The contour interval for $u$ is $2 \mathrm{~m} \mathrm{~s}^{-1}$ with the zero contour drawn heavy; $\theta$ contours are shown in black with contour interval $2 \mathrm{~K}$. The solution for the (a) below-mountaintop inversion and solutions for corresponding constant- $N$ soundings with stability obtained by (b) averaging the low-level stability and (c) making a bulk-method estimate of low-level stability.

Figs. $4 \mathrm{c}$ and $5 \mathrm{c}$. The nonlinearity parameter for this simulation,

$$
\epsilon_{B}=\frac{N_{B} h_{0}}{U}=1.66,
$$

is greater than that obtained using the averaging method. Apparent in the bulk method solution is that, along the centerline, the region of reversed flow is detached from the mountain surface (Fig. 4c). Inspection of the surface $u$ field (Fig. 5c) shows that the reversed flow only extends to the surface away from the centerline. In this simulation the extent of the region of reversed flow at the surface fluctuates slowly with time. 

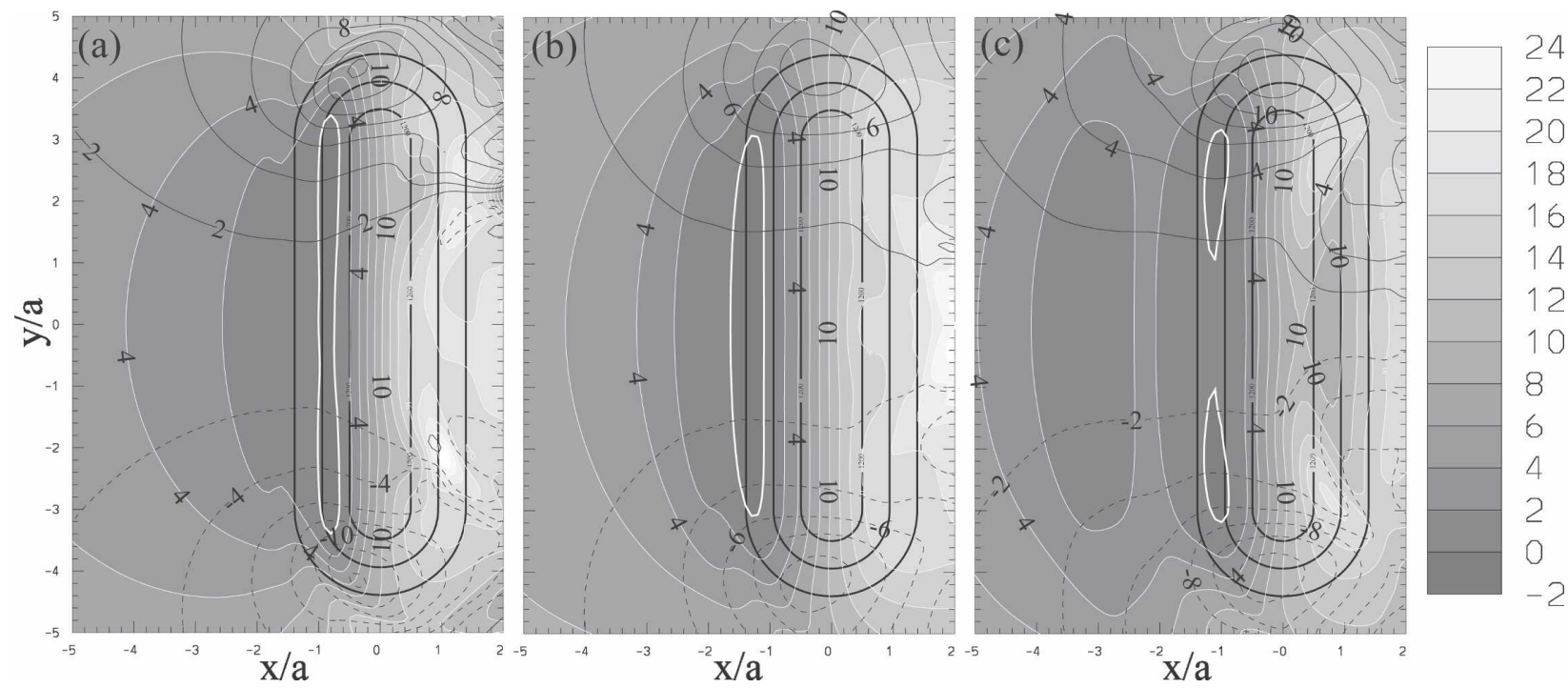

FIG. 5. The $u$ component of the wind at the surface shown in shading and white contours at $U t / a=25$ for a $h_{0}=1400 \mathrm{~m}, \beta=4$ mountain with upstream wind speed of $U=10 \mathrm{~m} \mathrm{~s}^{-1}$. The contour interval is $2 \mathrm{~m} \mathrm{~s}^{-1}$ and the zero contour is indicated by a heavy white line. The $v$ component is shown in black contours with a $2 \mathrm{~m} \mathrm{~s}^{-1}$ interval, the zero contour omitted. The case for the (a) belowmountaintop upstream inversion and corresponding constant- $N$ soundings obtained by (b) averaging and (c) making a bulk estimate of the upstream stability.

Also plotted in Fig. 5 is the $v$ component of surface wind in black contours. The magnitude of the $v$ component exceeds $2 \mathrm{~m} \mathrm{~s}^{-1}$ upstream of $x=-5 a$ in both the below-mountaintop inversion and bulk-methodestimated cases while the $2 \mathrm{~m} \mathrm{~s}^{-1}$ contour only extends upstream to $x=-4 a$ in the averaging case. This upstream extension of the cross-stream wind component acts to divert flow laterally around the mountain, as will be examined in more detail in the next section. Overall, the simulation using the averaging method better reproduces the flow structure in the elevated inversion simulation throughout the blocked region upstream of the mountain.

\section{b. Leeside structure}

Figures 4 and 5 also show significant differences downstream of the crest between the three cases. The averaging method produces stronger downslope winds than those produced by the original sounding with an upstream inversion, whereas the downslope winds generated in the bulk-estimate case are much weaker. The differences in the leeside structure are even more pronounced for flow over a slightly higher, 1600-m-high mountain. Figure 6 shows the vertical vorticity and horizontal wind vectors at the surface in the lee of both the $\beta=1$ and 4 mountains. Figures $6 a$ and $6 d$ show the reference solution when a mountaintop inversion is present in the upstream $\theta$ profile, clearly the wake region for the $\beta=4,1600-\mathrm{m}$ mountain is much larger than the $\beta=1$ case; however, the strength of the vertical vorticity is similar for both. For both mountain shapes, the wake structure in the solution with the bulk method estimated sounding (Figs. 6c and 6f) resembles the mountaintop inversion case much better than that for the averaging method (Figs. $6 \mathrm{~b}$ and $6 \mathrm{e}$ ). Indeed, there is only a very weak wake in the averaging-method solutions. The bulk method leads to a stronger wake because $N_{B}$ exceeds $N_{A}$ and thereby maps the flow with the upstream inversion to a constant-stability flow with larger nonlinear parameter $\left(\epsilon_{\mathrm{B}}=1.98\right.$ in comparison to $\left.\epsilon_{\mathrm{A}}=1.22\right)$.

\section{Parameter space mappings}

We now turn our attention from a few representative cases to the behavior over a wider range of parameter space. As discussed earlier, the CUS model is described by two parameters: $\epsilon$, the mountain height scaled by the vertical wavelength of a linear hydrostatic mountain wave, and $\beta$, the ratio of cross-stream to along-stream mountain scales.

\section{a. Windward flow deceleration}

\section{1) Minimum wind, in actual CUS conditions}

Smith and Grønås (1993) determined the minimum surface wind speed on the windward slope as a function of $\epsilon$ in series of nonlinear numerical simulations but, 

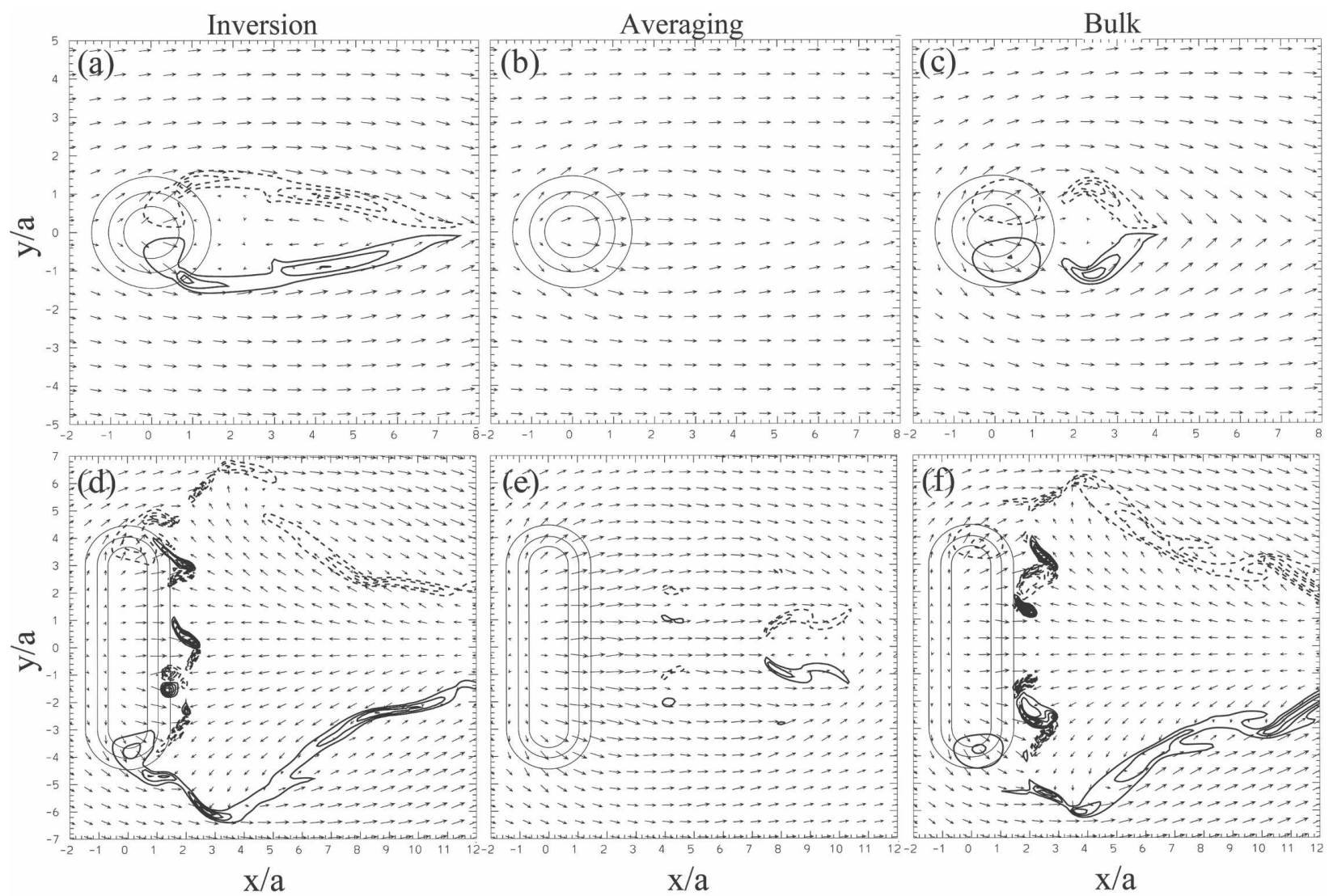

FIG. 6. The solution for uniform flow impinging on an $h_{0}=1600-\mathrm{m}$ mountain at $U t / a=25$ for a (a)-(c) $\beta=1$ mountain and (d)-(f) $\beta=4$ mountain. Solutions are (a) and (d) for the mountain top inversion, (b) and (e) for constant upstream stability obtained by the averaging method, and (c) and (f) for constant upstream stability obtained by the bulk method. Contours show the surface vertical vorticity with a contour interval of $0.002 \mathrm{~s}^{-1}$, no zero contour (solid and dashed lines represent positive and negative values, respectively). Horizontal wind vectors at the surface are also shown.

since their primary focus was on the flow field prior to wave breaking, the results do not extend to the large values of $\epsilon$ required in this study. Figure 7 shows our estimate of the normalized minimum surface wind speed on the windward slope $u_{m} / U$ as a function of $\epsilon$ for $\beta=1,2$, and 4 . The data in Fig. 7 were obtained from numerical solutions in which $h_{0}$ was systematically increased while $N$ was set to one of four possible values. The four different values of $N$ used in these experiments are the pairs $N_{A}$ and $N_{\mathrm{B}}$, calculated from (1) and (2) for the below-mountaintop inversion and mountaintop inversion soundings (see Fig. 1). The range of $\epsilon$ for an individual family of simulations with identical values of $N$ and different mountain heights overlaps the range of $\epsilon$ for other families so that the extent to which all the results for a given mountain shape collapse onto the same curve is an empirical measure of the universality of our result. As apparent in Fig. 7, all simulation results for a given $\beta$ are closely fit by a single curve, indicating that similar values of $\epsilon$, with different values
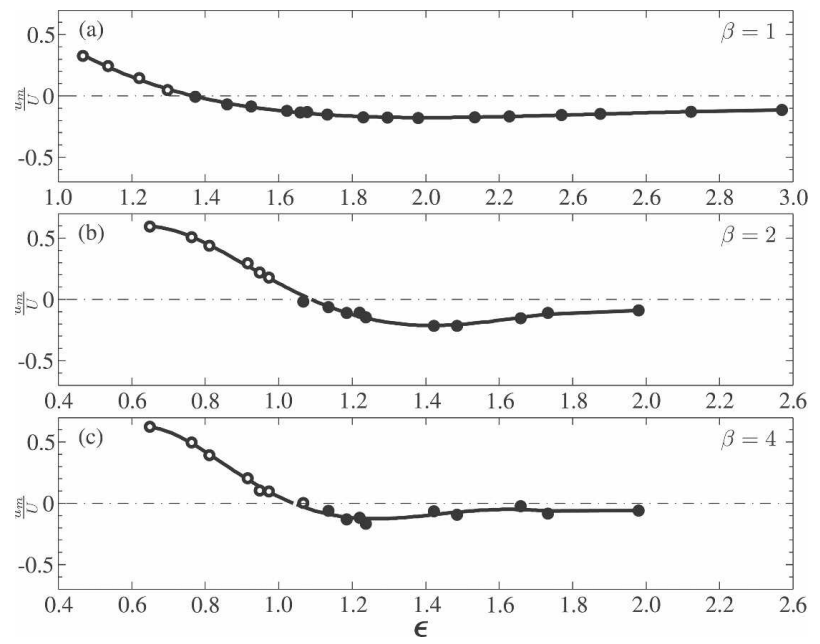

FIG. 7. Minimum windward-side $u$ velocity with constant upstream stability at a nondimensional time of $U t / a=25$ for flow over a (a) $\beta=1$, (b) $\beta=2$, and (c) $\beta=4$ mountain. Filled points indicated reversed flow. Also plotted is the fifth-order polynomial that fits the data in a least squares sense. 


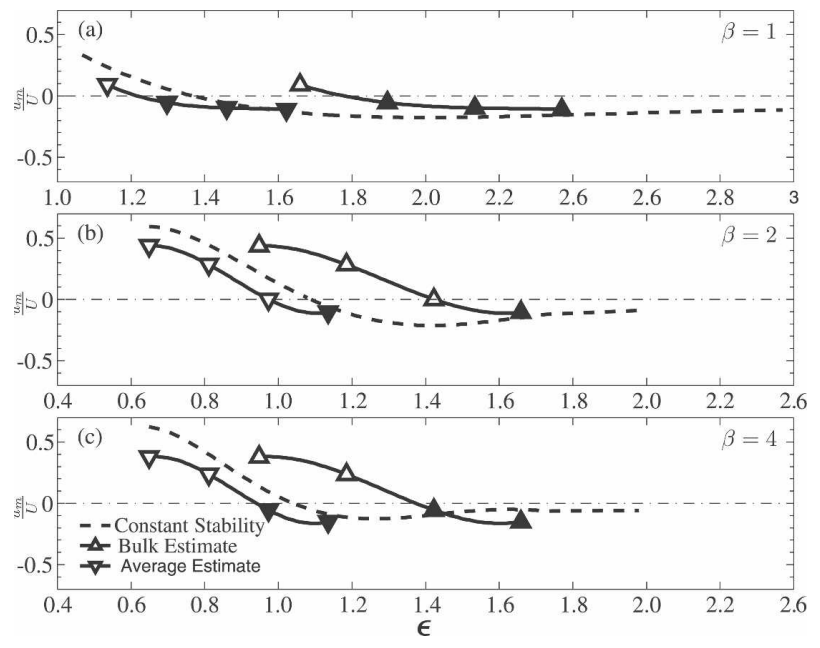

FIG. 8. Minimum $u$ component of wind on the upstream side of the ridge as a function of $\epsilon$ at $U t / a=25$. The curves are for the constant-stability upstream sounding (dashed), the upstream below-mountaintop inversion with the averaging-method estimate of stability (downward triangles), and the upstream belowmountaintop inversion with the bulk-method estimate of stability (upward triangles) for a (a) $\beta=1$, (b) $\beta=2$, and (c) $\beta=4$ mountain. Filled triangles indicate cases for which the flow reverses on the windward slope.

of $N$ and $h_{0}$, give similar results. The small degree of scatter about the best-fit curve is primarily due to a slight nonsteadiness in the upstream flow at $U t / a=25$, which was manifest as a small oscillation in the strength of the minimum wind. The phase of this oscillation varied among the various simulations, leading to a small amount of scatter about the best-fit curves. The strength of the oscillations, and the degree of scatter, increase with $\beta$.

\section{2) Applying the CUS model to cases with INVERSIONS}

The minimum wind speed on the windward slope found in the CUS theory (Fig. 7) is compared with the minimum wind speed from the below-mountaintop inversion and mountaintop inversion solutions in Figs. 8 and 9 , respectively. Estimates of $\epsilon \approx \epsilon_{\mathrm{A}}$ using (1) are depicted with downward-pointing triangles, whereas estimates $\epsilon \approx \epsilon_{\mathrm{B}}$ using (2) are indicated with upwardpointing triangles. Best-fit third-order least squares polynomials are also plotted for the $\epsilon_{\mathrm{A}}$ and $\epsilon_{\mathrm{B}}$ datasets.

If either parameter space $\left(\epsilon_{\mathrm{A}}, \beta\right)$ or $\left(\epsilon_{\mathrm{B}}, \beta\right)$ perfectly mapped the dependence of $u_{m} / U$ as a function of mountain height onto the predictions of CUS theory, then the data points for that mapping should coincide with the dashed curves in Figs. 8 and 9. Although neither approach is perfect, the averaging method clearly
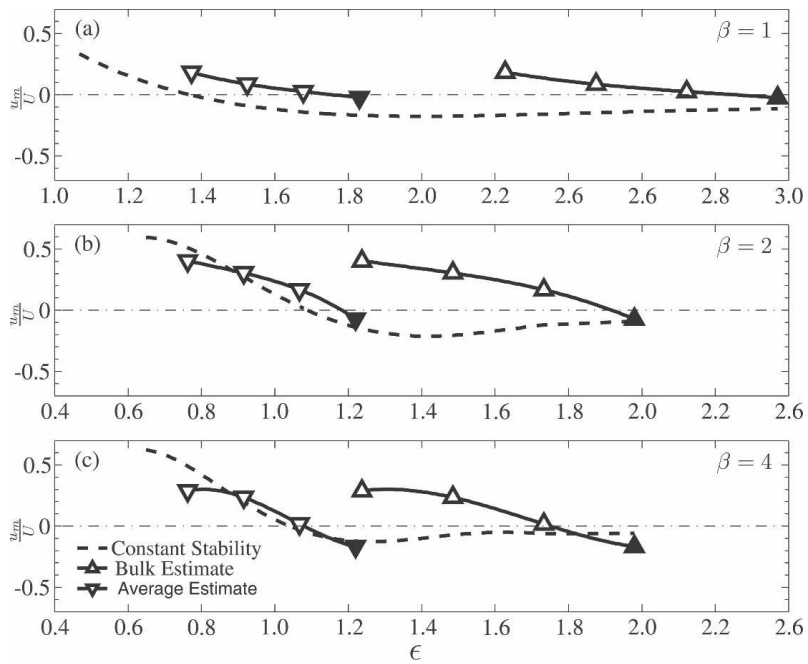

FIG. 9. As in Fig. 8 except for the mountaintop inversion.

works better than the bulk method. The bulk-method estimate of the stability, $N_{B}$, is larger than the $N_{A}$ obtained using the averaging method and, as consequence, the curves plotted using $\epsilon_{\mathrm{B}}$ lie to the right of those plotted using $\epsilon_{\mathrm{A}}$.

The averaging method works best when $\beta=2$ or $\beta=$ 4 and the inversion is at mountaintop level. For example, when $\beta=4, h_{0}=1200 \mathrm{~m}$, and the inversion is at mountaintop level, the minimum wind speed on the upstream slope is approximately $0.2 U$. The averaging method of $\epsilon_{A}$ for this case is about 0.9 , whereas the bulk-method estimate is roughly $\epsilon_{B}=1.5$. The minimum wind speed in a true CUS solution with $\epsilon=0.9$, corresponding to the averaged value, is about $0.2 \mathrm{U}$, agreeing with the actual numerical result for the case with the elevated inversion. On the other hand, when $\epsilon=1.5$, corresponding to the bulk method, the minimum wind speed in a true CUS solution is about $-0.1 U$, which is significantly decelerated from the winds that actually occur in the elevated inversion case.

The averaging and bulk estimates are further tested by considering their performance using inversions of different thicknesses and different elevations relative to the height of the peak Figs. 10a, 10b, and 10c show the minimum wind speed solution for a $\beta=2$ mountain when the inversion thickness is fixed at 125, 250, and $500 \mathrm{~m}$, respectively. The dashed lines in Fig. 10 represent an inversion centered at $600 \mathrm{~m}$ (Fig. 2a), the solid lines are for an inversion centered at $1000 \mathrm{~m}$ (Fig. 2b), and the dash-dot lines indicate a 1400-m-high inversion (Fig. 2c). The bulk estimate, $\epsilon \approx \epsilon_{B}$, shows a large sensitivity to the height and thickness of the inversion, whereas the values of $\epsilon \approx \epsilon_{A}$ obtained with the averaging method vary little and remain close to the solu- 


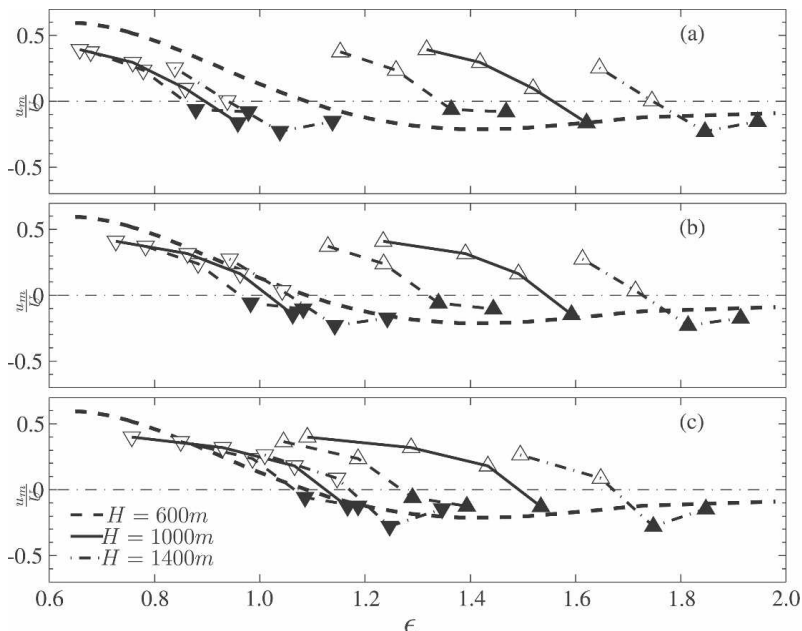

FIG. 10. Minimum $u$ component of wind on the upstream side of the $\beta=2$ ridge as a function of $\epsilon$ at $U t / a=25$. The inversion thickness is (a) $125 \mathrm{~m}$, (b) $250 \mathrm{~m}$, and (c) $500 \mathrm{~m}$. The curves represent the solution for the constant-stability upstream sounding (heavy dashed), an inversion centered at $600 \mathrm{~m}$ (thin dashed), an inversion centered at $1000 \mathrm{~m}$ (thin solid), and an inversion centered at $1400 \mathrm{~m}$ (thin dash-dot). The upward- (downward-) pointing triangles are the actual data points obtained using the averaging (bulk) estimate of the stability.

tion from the CUS theory. Not surprisingly, the averaging method performs worst when the inversion is thinnest (125 m, Fig. 2a). When the same increase in $\theta$ is spread across the wider inversions (Figs. 2b and 2c), the profile of $N$ is more nearly constant with height, and the averaging method gives better results. Interestingly, the bulk method only begins to give adequate results in the case with the thickest, lowest inversion (the thickest inversion in the profiles in Fig. 2a). In summary, the averaging method gives the best estimate of the minimum wind speed on the upstream slope.

\section{3) Stagnation on the Windward SLOPE}

Related to the minimum windward-side wind speed is the presence of flow reversal on the windward slope. Windward side stagnation is synonymous with an isentropic surface being pierced by the mountain surface (Smith 1988). The critical parameter $\hat{\epsilon}$ at which stagnation first occurs is given by the roots of the curves shown in Fig. 7.

Plotted in Fig. 11 is the relative error between the critical $\epsilon$ for stagnation in the constant-stability cases and the averaging or bulk estimates of the critical estimate of $\epsilon$ in the inversion cases. The downwardpointing triangles and upward-pointing triangles in Fig. 11 are

$$
\Delta S_{A}=\frac{\hat{\epsilon}_{A}-\hat{\epsilon}}{\hat{\epsilon}} ; \Delta S_{B}=\frac{\hat{\epsilon}_{B}-\hat{\epsilon}}{\hat{\epsilon}}
$$
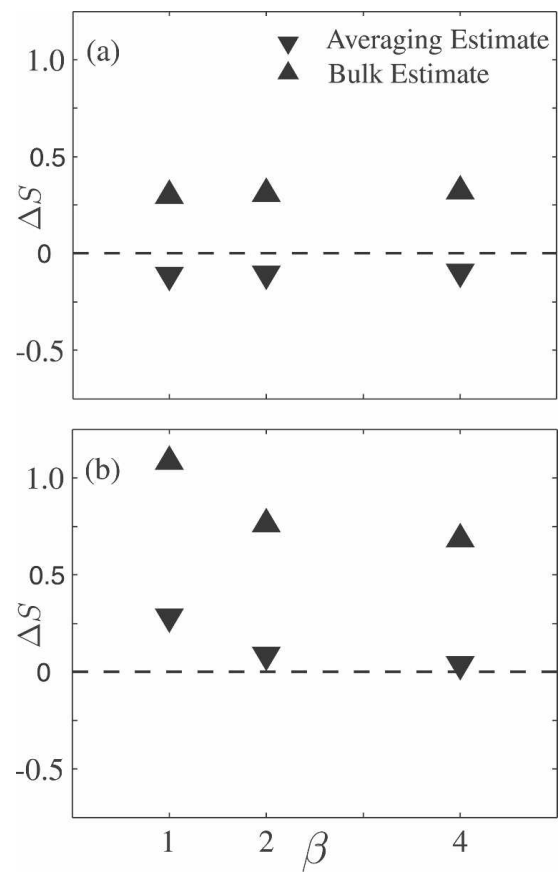

FIG. 11. The relative difference between the critical $\epsilon$ for stagnation in the constant-stability cases and the critical values of $\epsilon$ from the averaging (downward-pointing triangles) or bulk (upward-pointing triangles) estimated from a sounding with an upstream inversion: (a) below-mountaintop inversion and (b) an inversion at the mountain top.

respectively. Here $\hat{\epsilon}_{A}$ and $\hat{\epsilon}_{B}$ are the estimates of $\epsilon$ at stagnation using (1) and (2), respectively, to estimate the low-level stability when there is an inversion and $\hat{\epsilon}$ is the critical $\epsilon$ at stagnation in the CUS theory.

Consistent with the previously discussed prediction of the minimum wind speed, the averaging method gives better predictions than the bulk method for the critical $\epsilon$ for stagnation. Note that the relative errors for both the averaging and bulk methods are relatively independent of $\beta$, suggesting that the dependence of the stagnation point on $\beta$ in the CUS theory is unaffected by the presence of an inversion.

\section{b. Low-level flow diversion}

While the flow deceleration and development of reversed flow on the windward slope is of interest in its own right, in many practical applications estimates of $N h_{0} / U$ are used to assess the extent to which the lowlevel flow is directed around, instead of over, an obstacle (e.g., Chen and Smith 1987). In the following we will asses the extent to which the CUS theory can be used to estimate the degree of flow diversion when inversions are present in the upstream flow. The lowlevel flow diversion is calculated using the control volume shown in Fig. 12. The volume extends from $x=0$ 


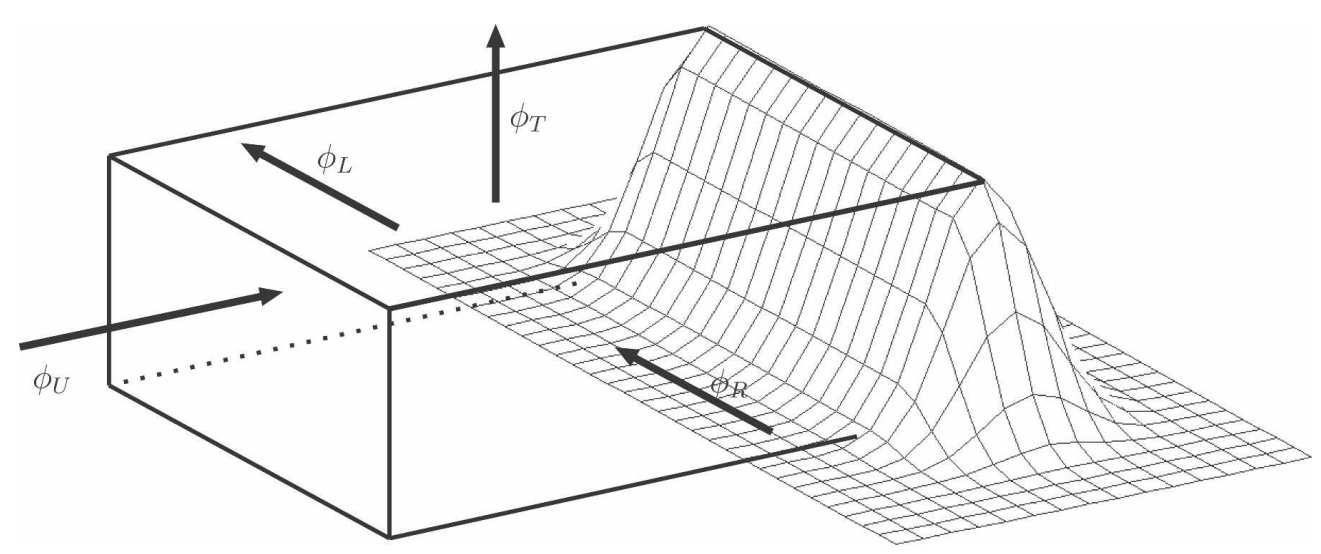

FIG. 12. Schematic of the control volume for the low-level flow diversion calculation.

at the ridge crest to $x=-12 a$ at the upstream inflow boundary (and uses data on the second grid to extend the volume well upstream of the mountain). The lateral sides of the volume extend upstream from the edge of the uniform-height section of the ridge crest at $y=$ $\pm(\beta-1) a$. The top of the control volume is a level surface located at $z=h_{0}$. If $\phi_{U}, \phi_{R}, \phi_{L}$, and $\phi_{T}$ are the integrated mass fluxes over each surface shown in Fig. 12 , then mass conservation requires

$$
\phi_{U}=\left(\phi_{L}-\phi_{R}\right)+\phi_{T} .
$$

Assuming flow through the upstream face is the sole source of mass into the volume (since it dominates the mass entering the volume through any of the other faces), the flow diversion may be defined as the ratio

$$
\mathrm{FD}=\frac{\phi_{L}-\phi_{R}}{\phi_{U}}
$$

Since the uniform-ridge section of the $\beta=1$ mountain has zero width, this calculation was done only for $\beta=$ 2 and 4.

\section{1) FLOW DIVERSION WITH UNIFORM UPSTREAM STABILITY}

The flow diversion as a function of $\epsilon$ for the true CUS model is shown in Fig. 13 for the $\beta=2$ and 4 mountains. This estimate was constructed using the same overlapping families of simulations that produced the data for Fig. 7 and, as before, a "universal" least squares fifth-order polynomial was fit to the data points. Figure 13 shows that the resulting polynomials fit the data very well for both the $\beta=2$ and 4 mountains.

\section{2) Comparison with the INVERsion CASE}

Figure 14 shows how the averaging and bulk estimates of the flow diversion for the below-mountaintop
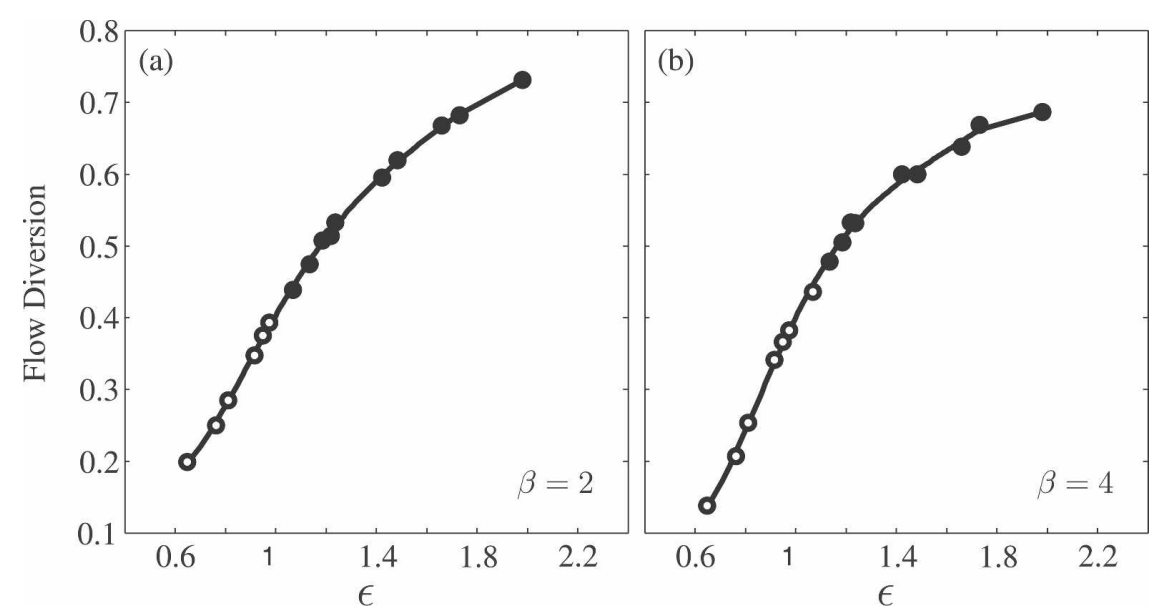

FIG. 13. Flow diversion for the (a) $\beta=2$ and (b) $\beta=4$ mountains when the stability is constant upstream. The curve is the least squares best-fit fifth-order polynomial. Reversed flow is indicated by filled data points while nonreversed flow is indicated by open data points. 


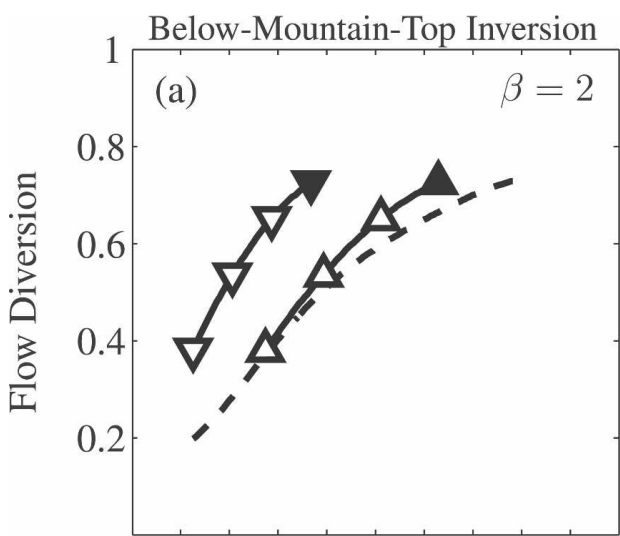

Mountain-Top Inversion
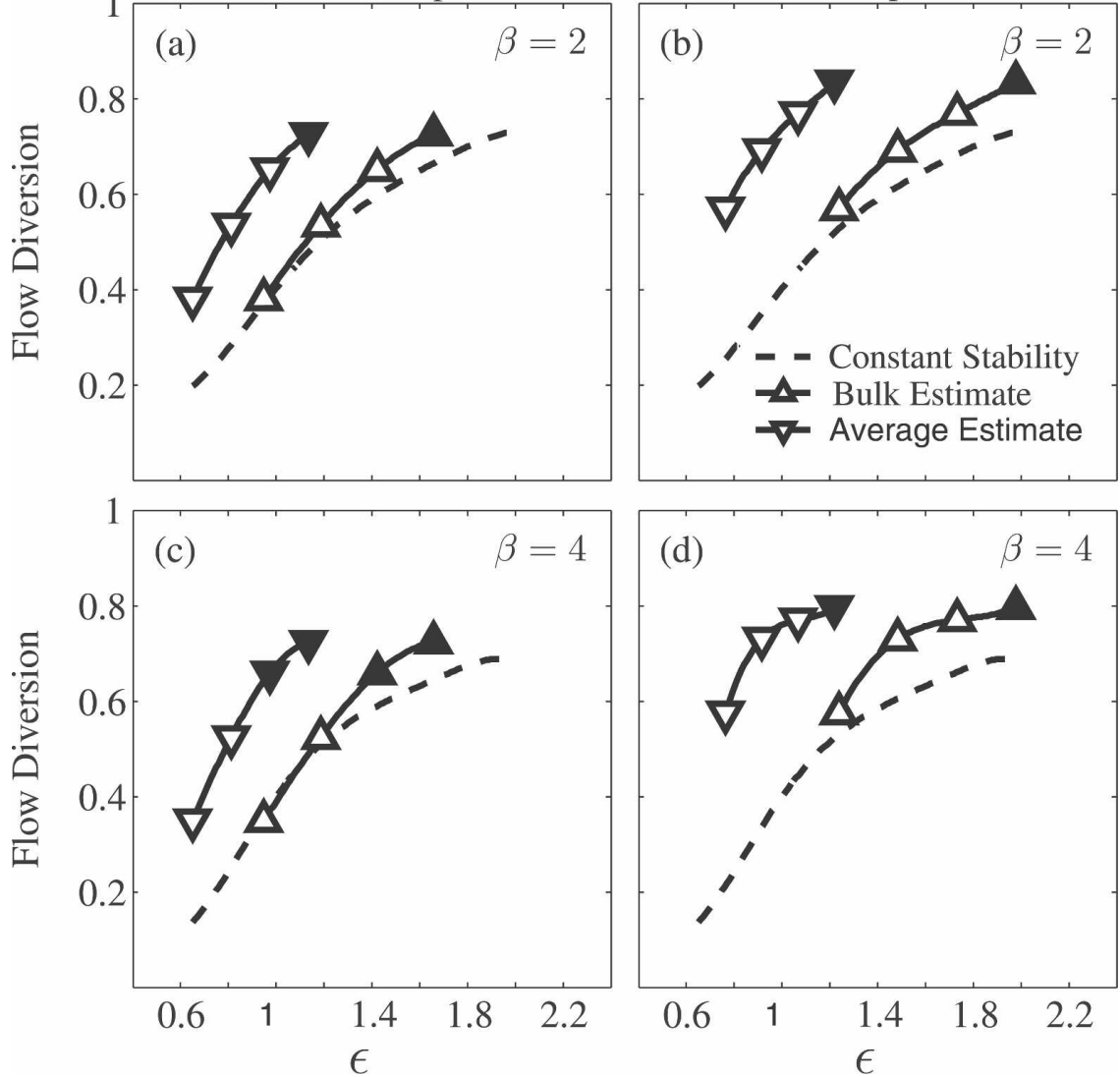

FIG. 14. Flow diversion around the mountain as a function of $\epsilon$ at $U t / a=25$ for a $\beta=2$ mountain with the (a) below-mountaintop inversion and (b) mountaintop inversion and a $\beta=4$ (c) below-mountaintop inversion and (d) mountaintop inversion. The upwind- and downward-pointing triangles indicate the low-level stability was estimated with the bulk and averaging methods, respectively. The curve is the best-fit least squares fifth-order polynomial of the data. The flow diversion for the CUS theory is shown with a dashed line for reference.

inversion and mountaintop inversion cases compare with the $\epsilon$ dependence of the true CUS solution. The downward-pointing triangles indicate data points for which the low-level stability was estimated using the averaging method, while the upward-pointing triangles indicate that the low-level stability was estimated using the bulk method. Filled triangles indicate the presence of reversed flow on the upwind slope. Each set of triangles is connected by a solid curve representing the least squares third-order polynomial fit of the data. The CUS curves from Fig. 13 are also plotted as dashed lines in Fig. 14 for reference.

As apparent in Fig. 14, the bulk method is a better predictor of the low-level flow diversion. The bulk method allows an especially good estimate of the flow diversion in the below-mountaintop inversion simulations. The degree to which the bulk method correctly maps the flow diversion in solutions with elevated in- versions onto the true CUS behavior decreases with increasing $\epsilon$.

The averaging and bulk estimates were further tested with the additional combination of soundings and mountain heights indicated in Fig. 2 (i.e., the same simulations used to construct the minimum wind speed curves in Fig. 10). In these additional tests (not shown), the bulk method gave the best prediction of low-level flow diversion and showed little sensitivity to height and thickness of the inversion, while the averaging method gave poorer results.

Although the averaging method is superior to the bulk method for estimating the minimum wind speed from the constant stratification model, it is inferior for the estimation of flow diversion. The superiority of the bulk method is linked to the upstream distribution of the $y$-component velocity perturbations. As discussed in connection with Fig. 5, when constant wind speed 
and stability simulations are performed with $N=N_{A}, v$ perturbations in excess of $2 \mathrm{~m} \mathrm{~s}^{-1}$ stop at $x=-4 a$, whereas they extend well past the upstream boundary of the fine mesh when either $N=N_{B}$ or an elevated inversion is present. Since the $y$ component of the wind controls the mass flux through the lateral boundaries of the control volume, it is not surprising that the flow diversion is better approximated by the bulk method.

\section{Sensitivity to $N$ aloft}

To investigate the sensitivity of our solutions to variations in stability aloft, a series of simulations were conducted in which the Brunt-Väisälä frequency above the inversion was halved $\left(N_{U}=0.005 \mathrm{~s}^{-1}\right)$ and doubled $\left(N_{U}=0.020 \mathrm{~s}^{-1}\right)$ from the value in the previously described simulations $\left(N_{U}=0.010 \mathrm{~s}^{-1}\right)$. This range of $N$ is much greater than the actual variation in the average $N$ throughout any deep layer in the earth's middle and upper troposphere. The stability within and below the inversion was left unchanged and was identical to that in the mountaintop inversion cases for the previously investigated mountain heights of $h_{0}=1000,1200,1400$, and $1600 \mathrm{~m}$. The $\beta=2$ simulations were then repeated using these higher and lower values of upper-level stability.

The variations in the low-level flow structure upstream of the mountain were generally quite insensitive to these changes in the upper-level stability. This is illustrated in Fig. 15, which shows a vertical cross section along the centerline of the $1600-\mathrm{m}$ mountain for the three different values of $N_{U}$. In each case, the winds are slightly reversed over a similar region above the upper portion of the windward slope (inside the heavy white contour). The upwind extent of the 2, 4, and 6 $\mathrm{m} \mathrm{s}^{-1}$ isotachs among the three cases is also remarkably similar, although there is a slight increase in the size of the region of reversed flow in the case with the weakest stability aloft.

In contrast, much more significant sensitivities are seen in the shooting flow on the downstream side of the ridge; these appear to be related to changes in the wave breaking regions aloft. The strength and downstream extent of the wave breaking, and of the shooting flow, decreases with decreasing stability aloft. Changes in the stability aloft also influence the lee vortex structure such that the extrema in the surface vertical vorticity increase with increasing $N_{U}$, along with the spatial complexity of the wake geometry (not shown).

For a more quantitative evaluation of the influence of $N_{U}$ we return to the metrics previously used to describe flow blocking in section 4 . The three curves in Fig. 16a show the normalized minimum surface wind
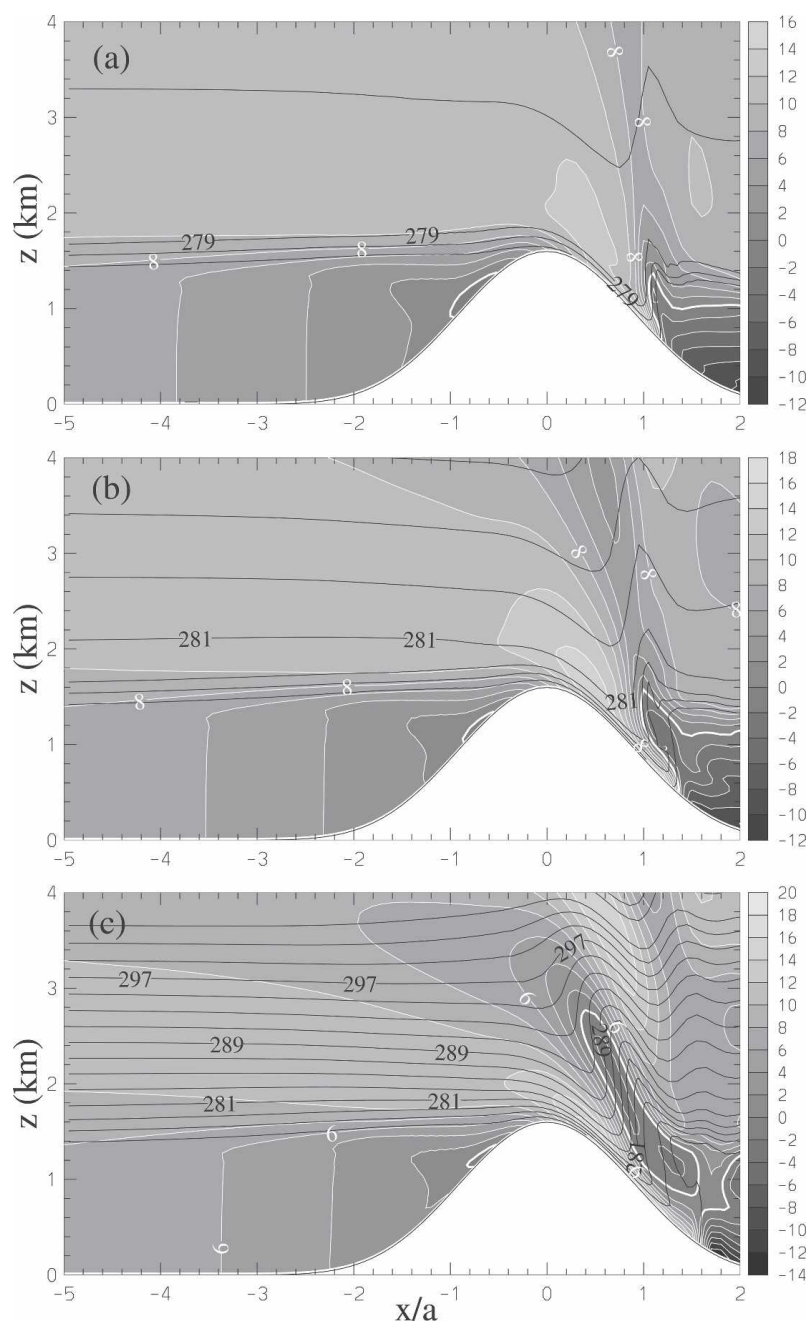

FIG. 15. The $u$ component of the wind along the centerline $y=$ 0 , shown in shading and white contours at $U t / a=25$ for an $h_{0}=$ $1600 \mathrm{~m}, \beta=2$ mountain and an upstream wind speed of $U=10$ $\mathrm{m} \mathrm{s}^{-1}$ with a mountaintop inversion. The contour interval for $u$ is $2 \mathrm{~m} \mathrm{~s}^{-1}$ with the zero contour drawn heavy; $\theta$ contours are shown in black with contour interval $2 \mathrm{~K}$. The Brunt-Väisälä frequency above the inversion is (a) $N=0.005 \mathrm{~s}^{-1}$, (b) $N=0.010 \mathrm{~s}^{-1}$, and (c) $N=0.020 \mathrm{~s}^{-1}$.

speed on the windward slope $\left(u_{m} / U\right)$ as a function of mountain height for each of the three values of $N_{U}$. With the exception of the 1400-m mountain, the dependence on $N_{U}$ is minimal, and therefore the sensitivity of the results shown in Fig. 9b to upper-level stability is also small.

Low-level flow diversion, defined by (5), is plotted as a function of mountain height for the three different values of $N_{U}$ in Fig. 16b. Flow diversion shows little dependence on the stability above the inversion, even for the 1400-m-high mountain, for which the sensitivity of the upstream flow deceleration to $N_{U}$ was greatest. 


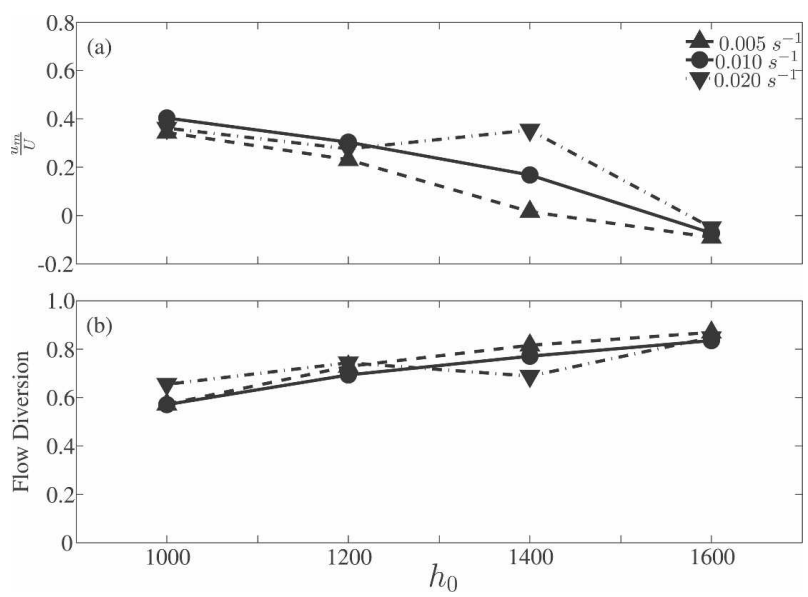

FIG. 16. The (a) minimum windward-side $u / U$ and (b) flow diversion as a function of $h_{0}$ for a $\beta=2$ mountain with a mountaintop inversion and upstream wind speed of $U=10 \mathrm{~m} \mathrm{~s}^{-1}$. The stability above the inversion varies between $N=0.005 \mathrm{~s}^{-1}$ (upward triangles), $N=0.010 \mathrm{~s}^{-1}$ (circles), and $N=0.020 \mathrm{~s}^{-1}$ (downward triangles).

As a consequence, the sensitivity of the flow diversion results shown in Fig. $14 \mathrm{~b}$ to $N_{U}$ is minimal.

\section{Conclusions}

When $N$ and $U$ are constant, a relatively simple parameter space controls the flow impinging upon an isolated mountain. The CUS theory is not, however, directly applicable in the real world where actual atmospheric conditions differ from the idealized model. Nevertheless, the simplicity of its parameter space has led many authors to apply results from CUS theory in real-world situations to estimate the basic morphology of the orographically modified flow. We have therefore conducted a series of numerical simulations with upstream inversions to assess the applicability of the CUS theory under more realistic conditions. The soundings considered represent a maximum realistic low-level perturbation to the uniform stability assumption in the constant stratification theory and, as such, a stringent but physically relevant test of each theory.

We considered two distinct indicators of low-level blocking: the minimum wind speed on the windward slope and the fraction of the incoming mass flux that deviates laterally around the mountain. Our results indicate that neither the averaging or the bulk method provides a single best technique to estimate the lowlevel stability for use with the constant-stratification theory. On one hand, the averaging method yields a value of $\epsilon$ for which the CUS solution more closely matches the upwind minimum wind speed of flows with elevated inversions (and the bulk method gives a larger value of $\epsilon$, for which the CUS solution exhibits too much flow deceleration). On the other hand, the value of $\epsilon$ obtained from the bulk method corresponds to a CUS solution matching the degree of flow diversion in the elevated inversion cases much better than the solution associated with the $\epsilon$ obtained by averaging (and the smaller value of $\epsilon$ given by the averaging method significantly underpredicts the mass actually deflected around the mountain in the elevated inversion cases). This suggests additional parameters must be considered to properly understand flow blocking when low-level variations in static stability are present upstream. However, one possible candidate, the stability above the inversion, had only a small effect on the minimum windward side wind speed and the flow diversion. In the end, the optimal method chosen to predict flow blocking depends on the desired application. To predict the onset of flow reversal the averaging method is the best method, but to predict low-level flow diversion the bulk method is best.

We have only considered the applicability of the CUS theory under variations in upstream static stability. Additional factors that influence the potential for flow blocking include surface friction, terrain shape, latent heating, and vertical variations in the low-level wind speed. The influence of variations in these parameters on the CUS theory remains unknown and is left for future research.

Acknowledgments. This research was supported by National Science Foundation Grants ATM-0242980 and ATM-0506589.

\section{REFERENCES}

Bauer, M. H., G. J. Mayr, I. Vergeiner, and H. Pichler, 2000: Strongly nonlinear flow over and around a three-dimensional mountain as a function of the horizontal aspect ratio. J. Atmos. Sci., 57, 3971-3991.

Bénech, B., E. Koffi, A. Druilhet, P. Durand, P. Bessemoulin, J. Campins, A. Jansa, and B. Terliuc, 1998: Dynamic characteristics of regional flows around the Pyrénées in view of the PYREX experiment. Part I: Analysis of the pressure and wind fields and experimental assessment of the applicability of the linear theory. J. Appl. Meteor., 37, 32-52.

Bougeault, P., 1983: A non-reflective upper boundary condition for limited-height hydrostatic models. Mon. Wea. Rev., 111, 420-429.

Bousquet, O., and B. F. Smull, 2003: Observations and impacts of upstream blocking during a widespread orographic precipitation event. Quart. J. Roy. Meteor. Soc., 129, 391-409.

Brinkmann, W. A. R., 1974: Strong downslope winds at Boulder, Colorado. Mon. Wea. Rev., 102, 592-602.

Chen, W.-D., and R. B. Smith, 1987: Blocking and deflection of airflow by the Alps. Mon. Wea. Rev., 115, 2578-2597.

Durran, D. R., 1999: Numerical Methods for Wave Equations in Geophysical Fluid Dynamics. Springer, 465 pp. 
and J. B. Klemp, 1983: A compressible model for the simulation of moist mountain waves. Mon. Wea. Rev., 111, 23412361.

Epifanio, C. C., and D. R. Durran, 2001: Three-dimensional effects in high-drag-state flows over long ridges. J. Atmos. Sci., 58, 1051-1065.

Georgelin, M., and E. Richard, 1996: Numerical simulation of flow diversion around the Pyrenees: A Tramontana case study. Mon. Wea. Rev., 124, 687-700.

Jiang, Q., J. D. Doyle, and R. B. Smith, 2005: Blocking, descent and gravity waves: Observations and modelling of a MAP northerly föhn event. Quart. J. Roy. Meteor. Soc., 131, 675701.

Kalthoff, N., and Coauthors, 2002: Mesoscale wind regimes in Chile at $30^{\circ}$ S. J. Appl. Meteor., 41, 953-970.

Klemp, J. B., and D. R. Durran, 1983: An upper boundary condition permitting internal gravity wave radiation in numerical mesoscale models. Mon. Wea. Rev., 111, 430-444.

LeVeque, R. J., 1996: High-resolution conservative algorithms for advection in incompressible flow. SIAM J. Numer. Anal., 33, 627-665.

Lilly, D. K., 1962: On the numerical simulation of buoyant convection. Tellus, 14, 148-172.

Manins, P. C., and B. L. Sawford, 1982: Mesoscale observations of upstream blocking. Quart. J. Roy. Meteor. Soc., 108, 427-434.

Marwitz, J. D., 1983: The kinematics of orographic airflow during Sierra storms. J. Atmos. Sci., 40, 1218-1227.

Mass, C. F., and G. K. Ferber, 1990: Surface pressure perturbations produced by an isolated mesoscale topographic barrier. Part I: General characteristics and dynamics. Mon. Wea. Rev., 118, 2579-2596.
Medina, S., and R. A. Houze, 2003: Air motions and precipitation growth in Alpine storms. Quart. J. Roy. Meteor. Soc., 129, 345-371.

Ólafsson, H., and P. Bougeault, 1996: Nonlinear flow past an elliptic mountain ridge. J. Atmos. Sci., 53, 2465-2489.

Overland, J. E., and N. A. Bond, 1995: Observations and scale analysis of coastal wind jets. Mon. Wea. Rev., 123, 2934-2941.

Rotunno, R., and R. Ferretti, 2001: Mechanisms of intense Alpine rainfall. J. Atmos. Sci., 58, 1732-1749.

Schär, C., and R. B. Smith, 1993: Shallow-water flow past isolated topography. Part I: Vorticity production and wake formation. J. Atmos. Sci., 50, 1373-1400.

Schumacher, P. N., D. J. Knight, and L. F. Bosart, 1996: Frontal interaction with the Appalachian Mountains. Part I: A climatology. Mon. Wea. Rev., 124, 2453-2468.

Smith, R. B., 1988: Linear theory of stratified flow past an isolated mountain in isosteric coordinates. J. Atmos. Sci., 45, 38893896.

_ 1989: Mountain-induced stagnation points in hydrostatic flow. Tellus, 41A, 270-274.

, and S. Grønås, 1993: Stagnation points and bifurcation in 3-D mountain airflow. Tellus, 45A, 28-43.

- S. Skubis, J. D. Doyle, A. S. Broad, C. Kiemle, and H. Volkert, 2002: Mountain waves over Mont Blanc: Influence of a stagnant boundary layer. J. Atmos. Sci., 59, 2073-2092.

Smolarkiewicz, P. K., and R. Rotunno, 1990: Low Froude number flow past three-dimensional obstacles. Part II: Upwind flow reversal zone. J. Atmos. Sci., 47, 1498-1511.

Spangler, T. C., 1987: Comparison of actual dividing-streamline heights to height predictions using the Froude number. $J$. Climate Appl. Meteor., 26, 204-207. 